\title{
Management of Obstructive Sleep Apnea with Oral Appliances: Our Experience
}

\section{B Jayan, BNBM Prasad, AK Rajput, R Bhattacharrya, RK Dhiman PS Thampi}

Respiratory Medicine, Orthodontics, Army Dental Centre, Army Hospital (R\&R)

\section{ABSTRACT}

Back ground : Obstructive sleep apnea results from any condition or disease that causes partial or complete obstruction of the air way when the patient assumes a supine position and goes to sleep. Oral appliances are viable options for treating patients with sleep disordered breathing.

Patient and methods: Oral appliances used in our series which included 19 patients were tongue retaining device (TRD), acrylic Herbst splint appliance and Karwetzky activator. Polysomnography was done on 10 cases with oral appliances. All cases were evaluated on Epworth Sleepiness Scale.

Results: $8(80 \%)$ patients showed significant reduction in $\mathrm{AHI}$ in addition to reduction / cessation of snoring and improvement on Epworth Sleepiness Scale. In one of the cases AHI increased and in another case AHI remained almost same but Sa O2\%decreased. In both these cases, oral appliances were withdrawn. In the other 9 cases who had not yet been subjected to PSG, after insertion of oral appliance 7 of the patients reported significant reduction / cessation of snoring and improvement on Epworth Sleepiness Scale. Karwetzky activator was found to be more patient friendly than other appliances used in the study.

Conclusion: Oral appliances therapy for OSA is non invasive, cost effective and beneficial to affected patients if desired efficacy is achieved. It greatly improves quality of life and cardio pulmonary health. We have observed short term therapeutic efficacy of oral appliances both clinically and by PSG studies..

Indian J Sleep Med 2006; 1.4, 204-207

\section{Introduction}

$\longrightarrow$ bstructive sleep apnea results from any condition or disease that causes partial or complete obstruction of air way when the patient assumes a supine position and goes to sleep . The basic mode of function of oral appliances is to prevent the tongue from approaching the posterior wall of the pharynx and causing an obstruction. Oral appliances used in this study were tongue retaining device

Address for Correspondence:

Lt Col B Jayan

Army Dental Centre (R\&R), Delhi Cantt

Email: jayan_deepa@rediffmail.com
(TRD) , acrylic Herbst splint appliance and Karwetsky activator ( fig 1,2,3). This study evaluates therapeutic and clinical efficacy of oral appliances in the management of obstructive sleep apnea based on our experience.

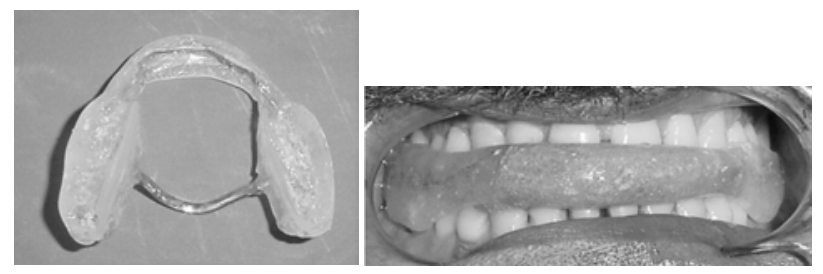

Fig 1: Tongue retaining device 


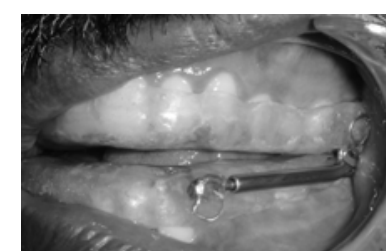

Fig 2: Acrylic Herbst splint appliance
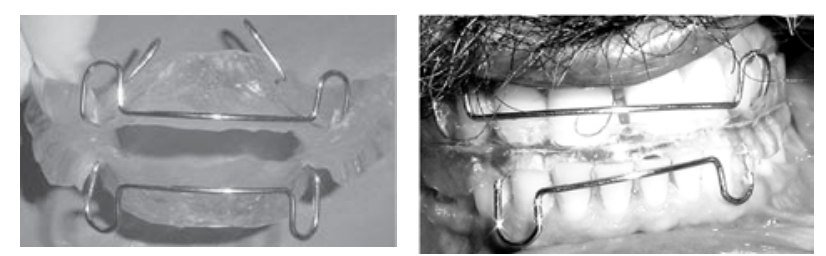

Fig 3: Karwetsky activator

\section{Patient and methods}

Oral appliances for OSA were prescribed to 19 patients of which 11 where males and 08 females and the mean age in years being 48 and 43.5 respectively. All these patients ware referred to us by Dept of Respiratory Medicine, Army Hospital (R\&R) Hospital after evaluating them clinically and with polysommographs (PSG) . Only mild, moderate and severe OSA cases who were not comfortable with nasal CPAP ( continuous positive air pressure) were taken up for oral appliances therapy . Case details were recorded on a sleep disordered breathing examination form which was inclusive of BMI ( body mass index), neck size, alcohol consumption and sedative usage details, sleep position, frequency and intensity of snoring, Epworth Sleepiness Scale, tongue size, air space evaluation between soft palate and tongue, periodontal and soft tissue examination, TM J evaluation, arch size, blood pressure history, maximum protrusive movement, clearance between central incisors at maximum opening. Various cephalometric readings were recorded and compared with established standards.Cephalograms was recorded at end expiration and patients were instructed not to swallow during radiography. Six cases each were treated with TRD and acrylic Herbst splint appliance respectively. Seven cases were treated with Karwetzky activator . Steps followed for appliance fabrication included impression making with sodium alginate impression material, preparation of working models, bite recording with modeling wax, articulation of models with recorded bite and appliance fabrication with self cure acrylic. During bite recording mandibular advancement did not exceed $70 \%$ of maximum protrusive movement. Vertical opening did not exceed 3 to $4 \mathrm{~mm}$ beyond free way space, 10 cases were subjected to PSG 8-12 weeks after insertion of oral appliances. All the 19 cases were also evaluated on Epworth Sleepiness Scale.

\section{Results}

Out of a total of 19 cases treated with oral appliances. 10 cases were subjected to PSG, 8-12 weeks after insertion of the appliance. Eight cases $(80 \%)$ showed significant reduction on AHI. Mean AHI decreased from 32.4 events per hours to 12.8 events per hour. 02 cases switched over from use of nasal CPAP to oral appliances. In two of the cases AHI increased and mean Sa O2 decreased. All the 17 cases were evaluated for subjective improvement on Epworth Sleepiness Scale and a mean decrease of 17.2 to 6.4 points was observed Subjective assessment of 17 cases reported reduction/cessation of snoring and reduced day a time sleepiness . Three patients, who were prescribed TRD, complained of inability to retain the tongue in tongue bulb for longer period. Five cases reported pain in TMJ region and headache during the first week and reported improvement in the same there after.

\section{Discussion}

Obstructive sleep apnea syndrome is a common disorder that is potentially fatal. It is characterized by repetitive episodes of complete or partial air way obstruction leading to diminished or absent air flow to lungs. These apneic /hypopneic spells last for 10-30 seconds. Common symptoms include loud snoring,excessive day time sleepiness, feeling of choking, restless sleep, unrefreshing sleep,changes in personality and nocturia. Less common symptoms are morning head aches, enuresis, reduced libido,spouse being worried about apneic spells, nocturnal sweating, noctural cough and symptomatic oesophageal reflux. Potential fatal illnesses associated with this disorder include hypertension, heart failure, nocturnal cardiac disarythimias, myocardial infarction and ischemic stroke[ 1].All cases in our series complained of snoring and excessive day time sleepiness. Two cases complained of morning head aches. Three cases each were diagnosed as hypothyroidism and coronary heart disease and were on medication for the same along with oral appliance therapy for OSA. 
The upper air way is a non rigid structure which includes hypopharynx, oropharynx and nasopharynx. During inspiration air pressure in the upper air way space becomes sub atmospheric caused by diaphargm attempting to pull air through the air way and the wall of the air way resists this air flow, The negative pressure tends to cause a change in shape of the air way which is resisted by the activity of tensor veli palatini and genioglossus muscles [2]. In OSA patients there is a reduction in the activity of those muscles that results in decreased air way space. In cases with obvious mandibular deficiency or functional retrusion, the tongue is placed posteriorly resulting in obstruction .Obesity can also narrow the upper airway. The mean BMI in our series were 27.8 and 26.9 for male and female respectively which are suggestive that most of them were obese. Maxillary deficiency can cause approximation of soft palate with posterior pharyngeal wall, thus reducing air way. Other common causes of obstruction includes adenoids and enlarged tonsils. Posterior and inferior placement of hyoid bone can also be a contributory factor. All cases of OSA snore but all snoring cases need not have OSA.

Dynamic MRI and CT scan are the useful imaging modality for snoring at sleep apnea patients. Lateral cephalogram, although providing only a two dimensional picture, is useful in examinations of upper air way, craniofacial and soft tissue analysis. We have subjected all our cases to lateral cephalograms and the most important measurement include posterior air way space, length of soft palate and distance of hyoid measured perpendicular to mandibular plane.

The treatment modalities include surgical procedures like maxillo -mandibular advancement, palato- uvulopharygoplasty, laser assisted uvulo palato plasty, hyoid suspension etc. Continuous positive air way pressure (CPAP) continues to be gold standard for treating the symptoms of OSA in both adult and children (4). CPAP suffers from poor patient compliances because of portability problems, pump noise, dryness of air way pressure and mask discomfort. In our series two cases switched over from CPAP therapy to oral appliance therapy.

An influential review of oral appliance therapy in 1995 for OSA accompanied by a practice parameter of the American Sleep Disorders Association signaled the entry of dentistry into mainstream sleep medicine. Adjustable mandible advancing oral appliances became the predominant form of dental therapy for sleep disordered breathing in 1990s. Controlled studies during late 1990s indicate effectiveness and greater patient preference for oral appliances compared to CPAP in mild and moderate OSA [7].

Oral appliances are indicated for use in patients with primary snoring, mild and moderate OSA who do not respond or not appropriate candidates for treatment with behavioral measures such as weight loss or sleep position change. In our series we prescribed oral appliances in two cases with severe OSA successfully. In one of the cases treated with acrylic Herbst appliance the AHI decreased from 75.2 to 48.3 and AI decreased from 51 to 8 [8].In another case a 52 year old female patient with severe OSA switched over from CPAP therapy to oral appliance therapy (Karwetzky activator).In this case AHI decreased from 43.6 to 17.9 ( Fig 4,5,6).

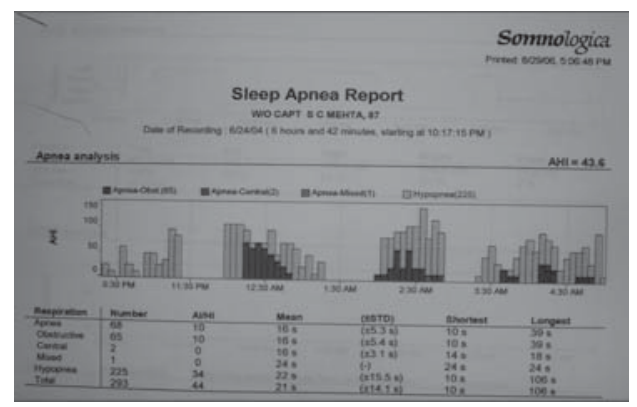

Fig 4: Diagnostic Polysomnograph of 52 year old female patient with severe OSA

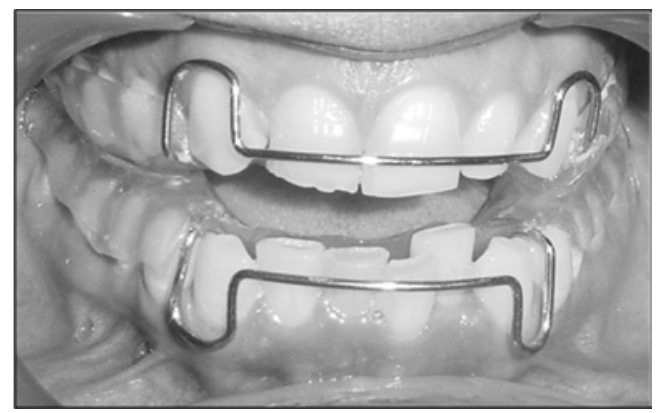

Fig 5: Patient with Karwetzky activator in situ 


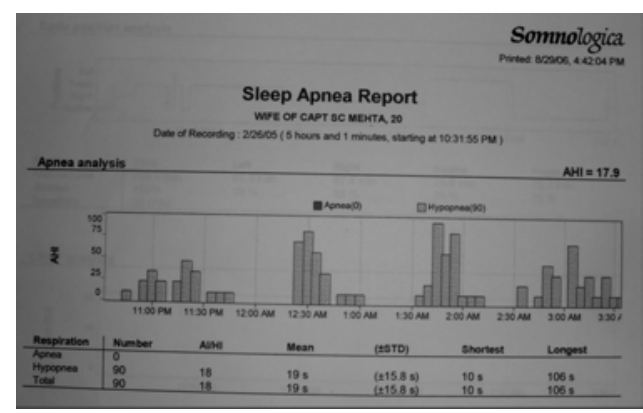

Fig 6: Polysomnograph recorded with oral appliance in situ

All the oral appliances work by placing the mandible forward and thus increasing the space between posterior pharyngeal wall and tongue. We used tongue retaining device, acrylic Herbst appliance and Karwetzky activator. All the appliances were made of hard acrylic. Most modern laboratories make appliances with thermoplastic materials which is more comfortable to patients. Titratable mandibular advancement device helps in slowly moving the mandible either anteriorly or posteriorly using the adjustable mechanism until successful results are achieved with minimum possible protrusive position. In our series we did not use these appliance designs; however we had to change the appliance frequently in four cases as it required increased mandibular advancement. So one had to fabricate the appliances repeatedly, this can be avoided if titratable appliances are used.In a recent investigation TAP (Thornston adjustable positioner), which is a titratable mandibular protrusive appliance, was examined for initial effect polysomnographically in patients with OSA. A predictable AHI based result was achieved. The authors also found the patient appliance acceptance was substiantially higher than with the Karwetzky activator[9]. We found Karwetsky activator most patient friendly . Some amount of titration is possible by adjusting the loops in this appliance. In a recent study Rose EC and coworkers have proven the initial efficacy of Karwetsky activator in the management of mild to moderate OSA. However it is unclear whether oral appliance treatment can be recommended as a life long option [10].

It is unclear as to why oral appliance was effective in most except in two of our cases. Various individual anatomic factors, the degree of vertical and saggital opening, the skeletal pattern of the skull and oropharyngeal tissue compliance may influence therapeutic efficacy. Similar such observations have also been reported in the literature [11].
We concluded that oral appliances may be an effective alternative for patients with mild to moderate OSA and severe cases not amenable to CPAP therapy. Such therapy, however, required regular polysomnographic follows ups. We have observed short term therapeutic efficacy of oral appliances both clinically and by PSG studies. We recommend long term studies to evaluate the continued efficacy of oral appliances in treating OSA.

\section{References}

1. Friedlander $\mathbf{A H}$ etal. Diagnosing and comanaging patients with obstructive sleep apnea syndrome. JADA Aug $2000 ; 131$. 1178-84.

2. Bailey DR . Oral evaluation and upper air way anatomy associated with snoring and obstructive sleep apnea. The Dental Clinics of North America . 2001 ; 45 (4) . 715-732.

3. Ivanhoe JR. Attansio R. Sleep disorders and oral devices. The Dental Clinics of North America. 2001 ; 45 (4). 73358

4. Ferguson KA, Takashsi O, Lowe AA et al ; A randomized cross over study of an oral appliance vs nasal continues positive air way pressure in the treatment of mild moderate sleep apnea. Chest ;1996.109; 1269 -75.

5. Holfstein V, Viner S, MateikaS : Treatment of obstructive sleep apnea with nasal continues positive air way pressure. Patient compliance, perception of benefits and side effect. Am Rev Respir Dis1992;145: 841-45.

6. Americans Sleep Disorders Association standards of practice committee, Practice parameters for the treatment of snoring and obstructive sleep apnea with oral appliances. Sleep; $1995.18: 511-513$.

7. Lowe AA : Dental appliances for the treatment of snoring and obstructive sleep apnea . In Kryger MH, RothT, DementW (Eds) : Principles and practice of sleep medicine, Ed 3. Philadephia , WB saunders . 2000; 929-39.

8. B Jayan, BNBM Prasad, AK Rajput, R Bhattacharya, RK Dhimman. Management of a case of severe obstructive sleep apnea with acrylic herbst splint appliance.Indian Journal of Sleep Medicine 2006;2(1). 157-60.

9. Rose EC, Germann M, Sorchter S, JonasIE. Case control study in the treatment of obstructive sleep disordered breathing with a mandibular protrusive appliance. J Orofac Orthop 2004; 65 : 489-500

10. Rose EC, Barthen GM, Staats R, Jonas IE ; Therapeutic efficacy of an oral appliance in the treatment of obstructive sleep apnea ; A 2 years follow up. Am J Orthod Dento facial Orthop 2002,121 (3) : 273-79

11. Isono S, Tanaka A, Tagaito Y, Sho Y, Nishino T. Pharyngeal patency on response to advancement in the mandible in obese anesthetized persons. Anesthesiology. 1997;87:1055-1062.

Indian Journal of Sleep Medicine (IJSM), Vol. 1, No. 4, 2006 